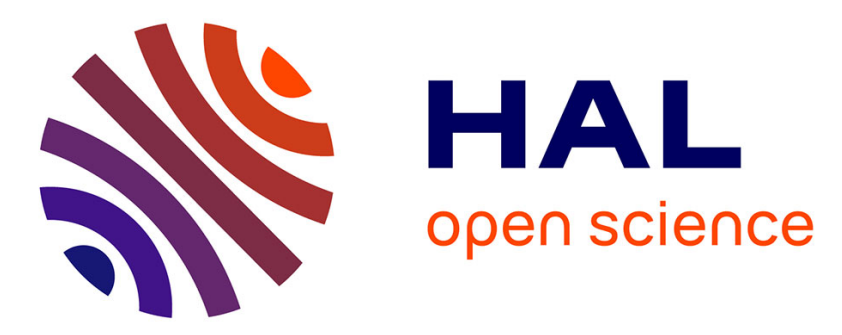

\title{
Comparaison de la qualité de vie de patients atteints d'atrophie multisystématisée et de maladie de Parkinson
}

Frédéric Torny, Mathieu Lacoste, Hélène Videaud, Marianne Chouly, M.-C. Parveau, Philippe Couratier

\section{- To cite this version:}

Frédéric Torny, Mathieu Lacoste, Hélène Videaud, Marianne Chouly, M.-C. Parveau, et al.. Comparaison de la qualité de vie de patients atteints d'atrophie multisystématisée et de maladie de Parkinson. Revue Neurologique, 2009, 10.1016/j.neurol.2009.02.001 . hal-00406542

HAL Id: hal-00406542

https://hal.science/hal-00406542

Submitted on 26 Aug 2020

HAL is a multi-disciplinary open access archive for the deposit and dissemination of scientific research documents, whether they are published or not. The documents may come from teaching and research institutions in France or abroad, or from public or private research centers.
L'archive ouverte pluridisciplinaire HAL, est destinée au dépôt et à la diffusion de documents scientifiques de niveau recherche, publiés ou non, émanant des établissements d'enseignement et de recherche français ou étrangers, des laboratoires publics ou privés. 


\title{
Comparaison de la qualité de vie de patients atteints d'atrophie multisystématisée et de maladie de Parkinson Comparison of quality of life in multiple system atrophy and Parkinson's disease
}

\author{
F. Torny ${ }^{a, b, *}$, M. Lacoste ${ }^{a, b}$, H. Videaud ${ }^{a}$, M. Chouly ${ }^{a}$, M.-C. Parveau ${ }^{a}$, P. Couratier ${ }^{a, b, c}$ \\ ${ }^{a}$ CMRR Limousin, 15, rue du Docteur-Raymond-Marcland, 87025 Limoges cedex, France \\ ${ }^{\mathrm{b}}$ Service de neurologie, CHU de Limoges, 2, avenue Martin-Luther-King, 87042 Limoges cedex, France \\ ${ }^{\mathrm{c}}$ EA 3174, faculté de médecine de Limoges, 2, rue du Docteur-Raymond-Marcland, 87025 Limoges cedex, France
}

R É S U M É

Introduction. - La qualité de vie (QDV) dans l'atrophie multisystématisée (AMS) est considérée plus altérée que celle de la maladie de Parkinson (MP). Le but de cette étude était de comparer la QDV chez dix patients atteints d'AMS probable (sous-type parkinsonien) et dix patients atteints de MP, appariés sur le handicap moteur.

Méthode. - Les patients inclus étaient ambulatoires et non déments. Les durées d'évolution dans le groupe AMS et MP étaient respectivement de 3,6 et 9,0 ans. Les patients étaient évalués sur la QDV, mesurée par le questionnaire SF-36 et une échelle visuelle analogique, l'état cognitif, l'apathie, la dépression, et les signes non moteurs.

Résultats. - La seule différence observée sur la QDV entre les deux groupes concernait la dimension vitalité de la SF-36, plus altérée chez les patients AMS et corrélée au score d'interférence du Stroop. La dépression et les signes non moteurs avaient un rôle délétère sur la QDV dans les deux groupes mais l'apathie et les difficultés au Stroop retentissaient uniquement sur la QDV des patients AMS.

Conclusion. - La QDV des patients AMS est comparable à celle des patients MP, appariés sur le handicap moteur, excepté sur la dimension vitalité plus altérée dans l'AMS et liée à la sensibilité à l'interférence.

\section{A B S T R A C T}

Purpose. - Quality of life (QoL) in multiple system atrophy (MSA) is thought to be poorer than in Parkinson's disease (PD), primarily because of motor impairment, autonomic dysfunction and depression. The aim of the study was to investigate QoL in 10 patients with probable MSA (parkinsonian subtype) compared with $10 \mathrm{PD}$ patients matched for motor disability on UPDRS III motor score.

\footnotetext{
* Auteur correspondant.

Adresse e-mail : frederic.torny@chu-limoges.fr (F. Torny).
} 
Methods. - All patients were ambulatory and non-demented. Mean durations of disease in MSA and PD patients were respectively 3.6 and 9.0 years. QoL was assessed using the SF-36 healthrelated questionnaire and a life satisfaction visual analogue scale. Patients were also evaluated for cognitive function (Mattis Dementia Rating Scale [Mattis DRS], Wisconsin Card Sorting Test [WCST], Stroop, Fluencies), depression (Beck Depression Inventory-II [BDI-II]), apathy (Modified Apathy Evaluation Scale) and were screened for non-motor symptoms (NMS Quest).

Results. - The only difference in QoL between MSA and PD patients matched for motor disability was that the SF-36 vitality subscore was more impaired in MSA and negatively correlated with interference index on Stroop word colour testing. Depression and non-motor symptoms were associated with poorer QoL in both groups. Among MSA patients, cognitive impairment (Stroop interference index) and apathy also had a negative impact.

Conclusion. - There was no major difference in QoL between MSA and PD patients matched for motor disability with a disease duration about 5 years longer. The SF-36 vitality subscore was more impaired in MSA and associated with interference sensitivity.

\section{Introduction}

L'atrophie multisystématisée (AMS) est une maladie neurodégénérative apparentée à la maladie de Parkinson (MP). Elle se caractérise sur le plan clinique par une association plus ou moins sévère d'un syndrome parkinsonien, d'une dysautonomie, d'un syndrome pyramidal et cérébelleux. Il existe deux sous-types cliniques majeurs (Gilman et al., 1998) : l'AMS-P et l'AMS-C dont l'évolution est un peu plus lente. Le traitement est symptomatique mais la dopathérapie est moins efficace que dans la MP. Deux études se sont intéressées à la qualité de vie (QDV) des patients atteints d'AMS, démontrant l'impact négatif du handicap moteur, de la dysautonomie et de la dépression, avec des différences minimes entre les deux soustypes cliniques (Benrud-Larson et al., 2005 ; Schrag et al., 2006). La QDV dans l'AMS serait moins bonne que dans la MP (Schrag et al., 2006).

L'objectif de cette étude est de comparer la QDV de dix patients présentant une AMS-P et dix patients atteints de MP appariés sur le handicap moteur (score moteur UPDRS III) et d'analyser l'impact de l'état cognitif, de la dépression, de l'apathie et des signes non moteurs.

\section{Patients et méthodes}

\subsection{Critères d'inclusion}

Les patients ont été inclus de manière prospective par le biais de la consultation, en excluant les patients non ambulatoires, les patients présentant une aggravation ou une modification thérapeutique récente ( $<3$ mois) et les patients avec des comorbidités sévères pouvant interagir avec la QDV. De même, les patients déments (critères DSM IV) ont été exclus car leur appréciation de la QDV est discutable.

Le diagnostic d'AMS-P probable était posé sur les critères consensuels (Gilman et al., 1998) : les patients (sept hommes et trois femmes) présentaient une dysautonomie, un syndrome parkinsonien peu dopasensible et une ataxie cérébelleuse légère. Le diagnostic de MP était défini sur les critères de l'United Kingdom Parkinson's Disease Brain Bank (UKPDBB) (Hughes et al., 1992) : les patients (quatre hommes et six femmes) ne présentaient pas de complication motrice invalidante (dyskinésies légères, période de off moins de deux heures par jour) et les symptômes cliniques étaient essentiellement un syndrome akinétorigide. Les patients du groupe AMS-P étaient appariés aux patients du groupe MP sur le score de handicap moteur évalué par l'Unified Parkinson's diseases Rating Scale part III (UPDRS III) (Fahn et al., 1987) avec une différence maximale de deux points.

\section{2. Évaluation clinique}

Les patients bénéficiaient sous leur traitement habituel, d'une évaluation, réalisée le même jour, de la QDV, de l'état moteur, cognitif et psychiatrique (dépression et apathie), et des signes non moteurs.

\subsubsection{Qualité de vie}

La QDV liée à l'état de santé était mesurée par la SF-36 (McHorney et al., 1993), autoquestionnaire validé dans de nombreuses langues et pathologies, comprenant 36 items regroupés en huit dimensions et deux scores globaux. Les résultats ont été calculés avec les algorithmes normés, compris entre zéro et 100, les valeurs les plus élevées représentant une meilleure fonction.

Une évaluation globale de la QDV était quantifiée sur une échelle visuelle analogique (EVA) de $100 \mathrm{~mm}$; le patient situant sa QDV entre " très insatisfaisante " $(0)$ et " très satisfaisante " (100). Cette méthode, déjà utilisée dans une autre étude sur la QDV dans l'AMS (Benrud-Larson et al., 2005) permet d'avoir une évaluation plus globale que la SF-36.

\subsubsection{Autres évaluations}

Le handicap moteur était mesuré par l'UPDRS III (Fahn et al., 1987).

Le bilan cognitif comprenait une évaluation globale sur l'échelle de Mattis et des fonctions exécutives (fluences verbales littérales avec la lettre $P$, catégorielles, test des cartes de Wisconsin et test de Stroop à 100 items).

L'apathie était évaluée par le Modified apathy evaluation scale, autoquestionnaire de 14 items validé dans la MP (Starkstein et al., 1992) et permettant d'établir un score de sévérité compris entre 0 et 42 . 
La dépression était évaluée par la Beck depression inventory-II (BDI-II), hétéroquestionnaire de 21 questions appréciant les symptômes dépressifs, chaque réponse étant côtée de zéro à trois, avec un score total supérieur à 13 définissant un syndrome dépressif (Beck et al., 1996).

La présence de signes non moteurs était recherchée par le Non-motor symptoms questionnaire (NMS Quest), récemment développé dans la MP (Chaudhuri et al., 2006).

\subsection{Analyse statistique}

Les différences entre les deux groupes ont été analysées par le test de Student apparié. Les proportions ont été comparées par le test de $\mathrm{Khi}^{2}$. Les corrélations ont été réalisées par le test des rangs de Spearman. Le niveau de significativité des tests était déterminé par un $p<0,05$.

\section{Résultats}

\subsection{Caractéristiques des patients}

Les caractéristiques des patients sont rapportées dans le Tableau 1. Les deux groupes différaient par une durée d'évolution de la maladie plus longue d'environ cinq ans chez les patients MP par comparaison au groupe AMS $(9 \pm 4,1$ ans versus $3,6 \pm 2,0$ ans ; $p=0,003)$. Il n'était pas observé de différence entre les deux groupes sur la sévérité des scores cognitifs, de dépression et d'apathie. Cependant, les patients AMS se plaignaient plus au NMS Quest que les patients MP (exonération incomplète $: p=0,002$; sentiment de dépression : $p=0,001$; perte d'intérêt sexuel : $p=0,01$; difficultés sexuelles : $p=0,04$; chutes : $p<0,001 ;$ malaises au passage en orthostatisme $: p=0,02$ ).

\subsection{Qualité de vie}

Les scores de QDV sont rapportés dans le Tableau 2. La QDV des patients AMS ne différait pas de celle des patients MP, excepté sur la dimension "vitalité " de la SF-36, significativement plus altérée (score moyen : $36,0 \pm 6,2$ versus $42,0 \pm 4,5$; $p=0,01)$.

\subsection{Facteurs associés à une mauvaise qualité de vie}

La QDV des patients des deux groupes n'était pas corrélée à l'âge des patients, la durée de la maladie ni à la posologie de L-Dopa.

Tableau 1 - Caractéristiques démographiques et cliniques des patients - moyenne (écart-type). Demographical and clinical characteristics of the subjects groups - mean (S.D.).

\begin{tabular}{|c|c|c|c|}
\hline & Patients AMS & Patients MP & $p$ \\
\hline Sexe $(H / F)$ & $7 / 3$ & $4 / 6$ & \\
\hline Âge (années) & $63,7(5,6)$ & $66,7(6,6)$ & ns \\
\hline Min-max & $54-70$ & $55-76$ & \\
\hline Durée maladie (années) & $3,6(2,0)$ & $9,0(4,1)$ & 0,003 \\
\hline Min-max & $1-6$ & $3-16$ & \\
\hline Score UPDRS III & $23,7(5,1)$ & $24,7(8,4)$ & ns \\
\hline Min-max & $18-34$ & 14-39 & \\
\hline Score UMSARS II & $16,7(3,9)$ & - & \\
\hline Min-max & $11-23$ & & \\
\hline Score Mattis (/140) & $137,3(3,9)$ & $135,2(6,1)$ & ns \\
\hline Fluences littérales (lettre P /2 minutes) & $21,6(10,4)$ & $16,5(7,1)$ & ns \\
\hline Fluences catégorielles (animaux /2 minutes) & $24,2(7,8)$ & $23,8(7,9)$ & ns \\
\hline \multicolumn{4}{|l|}{ Wisconsin card sorting test } \\
\hline Nombre de catégories & $5,1(1,4)$ & $4,9(1,2)$ & ns \\
\hline Nombre d'erreurs & $7,8(8,5)$ & $7,7(5,2)$ & ns \\
\hline Nombre d'erreurs persévératives & $3,7(7,4)$ & $2,7(2,9)$ & ns \\
\hline \multicolumn{4}{|l|}{ Stroop word color test } \\
\hline Temps condition de base (minutes) & $72,2(16,3)$ & $76,3(16,2)$ & ns \\
\hline Temps condition d'interférence (minutes) & $147,3(51,3)$ & $180,7(78,4)$ & ns \\
\hline Indice d'interférence (minutes) & $75,1(37,1)$ & $104,4(75,0)$ & ns $(0,25)$ \\
\hline Nombre d'erreurs (condition d'interférence) & $8,2(11,2)$ & $5,2(7,3)$ & ns \\
\hline Score dépression (BDI-II) (/63) & $15,3(8,1)$ & $14,1(4,8)$ & ns \\
\hline Score apathie (Apathy evaluation scale /42) & $12,7(3,5)$ & $9,6(4,8)$ & ns \\
\hline Dose equivalent $\mathrm{L}$-Dopa $(\mathrm{mg})$ & $470(408)$ & $929(379)$ & ns \\
\hline Min-max & $0-1250$ & $300-1350$ & \\
\hline Traitement antidépresseur (nombre patients) & $5 / 10$ & $3 / 10$ & ns \\
\hline
\end{tabular}

BDI-II : Beck depression inventory-II ; UMSARS II : Unified Multiple System Atrophy Rating Scale part II ; UPDRS III : Unified Parkinson's diseases Rating Scale part III ; ns : non significatif. 
Tableau 2 - Comparaison des scores de qualité de vie (QDV) entre les patients atteints d'atrophie multisysté matisée (AMS) et de maladie de Parkinson (MP) - moyenne (écart-type).

Comparison between quality of life scores in patients with multiple system atrophy and Parkinson's disease - mean (S.D.).

\begin{tabular}{llll}
\hline & Patients AMS & Patients MP \\
\hline Évaluation globale (EVA : 0-100 mm) & $42,7(14,5)$ & $46,8(22,3)$ & \\
Dimensions de la SF-36 & & $42,5(6,6)$ & $\mathrm{ns}$ \\
Activité physique & $39,1(11,3)$ & $41,4(12,6)$ & $\mathrm{n}$ \\
Limitations dues à l'état physique & $44,2(8,9)$ & $39,0(6,4)$ & $\mathrm{ns}$ \\
Douleur physique & $44,2(11,7)$ & $36,8(7,3)$ & $\mathrm{ns}$ \\
Santé générale & $33,4(4,8)$ & $42,0(4,5)$ & $\mathrm{ns}$ \\
Vitalité & $36,0(6,2)$ & $38,2(12,9)$ & \\
Vie relationnelle & $39,2(12,0)$ & $44,8(12,1)$ & $\mathrm{ns}$ \\
Limitations dues à l'état émotionnel & $44,8(9,9)$ & $40,4(5,9)$ & $\mathrm{ns}$ \\
Santé psychique & $39,8(8,4)$ & $37,7(6,6)$ & $\mathrm{ns}$ \\
Score composite physique SF-36 & $37,6(9,4)$ & $42,7(8,3)$ \\
Score composite mental SF-36 & $41,3(10,6)$ & $\mathrm{ns}$ \\
\hline
\end{tabular}

EVA : échelle visuelle analogique ; ns : non significatif.

Chez les patients AMS, la QDV mesurée à la SF-36 était négativement corrélée au score de la dépression à la BDI $(r=-0,68 ; p=0,03)$, à l'apathie $(r=-0,75 ; p=0,01)$ et à la présence de certains signes non moteurs (hypersialorrhée : $r=-0,76 ; p=0,007$; difficultés de déglutition : $r=-0,74$; $p=0,01$; perte de poids : $r=-0,65 ; p=0,04$; plainte mnésique : $r=-0,65 ; p=0,04)$. La présence de signes dysautonomiques n'était pas corrélée à une mauvaise QDV. La dimension " vitalité » de la SF-36 était négativement corrélée avec l'index d'interférence au Stroop $(r=-0,74$; $p=0,01)$.

Chez les patients MP, la QDV mesurée à la SF-36 était négativement corrélée à la dépression $(r=-0,74 ; p<0,01)$ et à la présence de signes non moteurs (difficultés de déglutition : $r=-0,66 ; p=0,03$; nausées : $r=-0,86$; $p=0,0006$; constipation : $r=-0,73 ; p=0,01$; perte de poids : $r=-0,67 ; p=0,03$; périodes de dépression : $r=-0,92$; $p<0,0001$; périodes d'anxiété : $r=-0,73 ; p=0,01 ;$ malaises au passage en orthostatisme : $r=-0,68 ; p=0,03$; rêves agités : $r=-0,82 ; p=0,002)$. La dimension "vitalité " de la SF-36 était négativement corrélée à la dépression $(r=-0,85$; $p=0,0008)$.

L'évaluation globale de la QDV par EVA n'était corrélée à aucune évaluation dans les deux groupes de patients.

\section{Discussion}

Dans cette étude, les patients AMS appariés sur le handicap moteur aux patients MP présentaient une QDV relativement comparable à celle des patients MP dont la durée d'évolution était plus longue d'environ cinq ans. Les patients non ambulatoires ayant été exclus, il n'est cependant pas possible d'extrapoler ce résultat à un stade évolué de la maladie. Il serait très intéressant de réaliser une comparaison à un stade plus évolué de la maladie pour renforcer l'hypothèse que la QDV dépend surtout du handicap lié aux symptômes moteurs. Cependant, une durée d'évolution plus longue exposerait au risque d'inclure des patients parkinsoniens avec des troubles cognitifs pour lesquels l'évaluation de la QDV est discutable. La seule différence significative entre les deux groupes concernait la dimension « vitalité » de la SF-36, plus altérée dans l'AMS et négativement corrélée aux performances du Stroop. Plusieurs études ont étudié le profil cognitif dans l'AMS et la MP mais les différences sont généralement minimes et variables selon la méthodologie (Dujardin et al., 2003). Dans cette étude où les patients présentaient des évaluations cognitives et psychiatriques comparables, il a été retrouvé un impact plus important de l'apathie et des difficultés au Stroop chez les patients AMS que chez les patients MP. Il existe donc probablement des différences subtiles dans le profil des fonctions cognitives et de l'apathie entre ces deux maladies.

Les symptômes non moteurs les plus retrouvés dans le groupe AMS étaient les troubles dysautonomiques mais aussi les périodes de dépression. Cependant, les scores de dépression étant normaux et comparables dans les deux groupes et il est possible que cette plainte chez les patients AMS soit plutôt le reflet d'une labilité émotionnelle. Dans les deux groupes, les plaintes non motrices influant sur la QDV étaient essentiellement digestives mais il existait aussi de nombreuses autres plaintes chez les patients MP. En effet, chez ces patients évolués (neuf ans de maladie en moyenne), les signes non moteurs étaient fréquents et plus péniblement perçus car survenant après une période d'amélioration motrice nette et soutenue sous dopathérapie.

\section{Conclusion}

En conclusion, la QDV des patients AMS ne diffère pas de celle des patients MP quand ils sont appariés sur le handicap moteur. Les symptômes non moteurs et la dépression ont un impact négatif sur la QDV. Dans l'AMS, les troubles cognitifs légers semblent avoir aussi un impact, en particulier l'apathie et la sensibilité à l'interférence, mesurée par le Stroop. Des études complémentaires sur un effectif plus large de patients, à des stades différents, restent nécessaires pour confirmer ces résultats.

Conflits d'intérêts : pas de conflits d'intérêt à déclarer en lien avec l'article. 
developments in Parkinson's disease, 2. Florham Park: Macmillan Healthcare; 1987. p. 153-63.

Beck AT, Steer RA, Ball R, Ranieri WF. Comparison of Beck depression inventories -IA and -II in psychiatric outpatients. J Pers Assess 1996;67:588-97.

Chaudhuri KR, Martinez-Martin P, Schapira AH, Stocchi F, Sethi $\mathrm{K}$, Odin $\mathrm{P}$, et al. International multicenter pilot study of the first comprehensive self-completed nonmotor symptoms questionnaire for Parkinson's disease: the NMSQuest study. Mov Disord 2006;21:916-23.

Benrud-Larson LM, Sandroni P, Shrag A, Low PA. Depressive symptoms and life satisfaction in patients with multiple system atrophy. Mov Disord 2005;20:951-7.

Dujardin K, Defebvre L, Krystkowiak P, Degreef JF, Destee A. Executive function differences in multiple system atrophy and Parkinson's disease. Parkinsonism Relat Disord 2003;9:205-11.

Fahn S, Elton RL, The members of the UPDRS Development Committee. The unified Parkinson's disease rating scale. In: Fahn S, Marsden CD, Calne DB, Goldstein M, editors. Recent

ilman S, Low PA, Quinn N, Albanese A, Ben-Shlomo Y, Fowler CJ, et al. Consensus statement on the diagnosis of multiple system atrophy. J Auton Nerv Syst 1998;74:189-92.

Hughes AJ, Daniel SE, Kilford L, Lees AJ. Accuracy of clinical diagnosis of idiopathic Parkinson's disease: a clinicopathological study of 100 cases. J Neurol Neurosurg Psychiatry 1992;55:181-4.

McHorney CA, Ware Jr JE, Raczek AE. The MOS 36-item ShortForm health survey (SF-36): II. Psychometric and clinical tests of validity in measuring physical and mental health constructs. Med Care 1993;31:247-63.

Schrag A, Geser F, Stampfer-Kountchev M, Seppi K, Sawires M, Kollensperger $\mathrm{M}$, et al. Health-related quality of life in multiple system atrophy. Mov Disord 2006;21:809-15.

Starkstein SE, Mayberg HS, Preziosi TJ, Andrezejewski P, Leiguarda R, Robinson RG. Reliability, validity, and clinical correlates of apathy in Parkinson's disease. J Neuropsychiatry Clin Neurosci 1992;4: 134-9. 